\title{
Facile synthesis of novel functionalized 1,3-selenazoles
}

\author{
Violeta Kanapickaitė, Vytas Martynaitis and Algirdas Šačkus* \\ Institute of Synthetic Chemistry, Kaunas University of Technology, LT-50254 Kaunas, Lithuania \\ E-mail: algirdas.sackus@,ktu.lt
}

\begin{abstract}
The reaction of selenourea with diethyl 2,4-dibromo-3-oxoglutarate afforded ethyl 2-(2-amino-5ethoxycarbonyl-1,3-selenazol-4-yl)-2-bromoethanoate. Treatment of the latter with acylating agents and various nucleophiles gave a series of new 4,5-disubstituted 2-amino-1,3-selenazoles. All compounds were characterized spectroscopically. The crystal structure determination of ethyl 2-(2amino-5-ethoxycarbonyl-1,3-selenazol-4-yl)-2-bromoethanoate is reported.
\end{abstract}

Keywords: 2-Amino-1,3-selenazole, selenourea, Hantzsch reaction, heterocycles

\section{Introduction}

Considerable interest in the synthesis of 1,3-selenazole derivatives exists due to their potential for practical applications. Functionalized 1,3-selenazole moieties are present in many pharmacologically active substances. ${ }^{1}$ The prominent examples are a potent antiviral agent selenazofurin (2- $\beta$-ribofuranosyl-1,3-selenazole-4-carboxamide) $\mathbf{1},{ }^{2}$ and a histamine $\mathrm{H}_{2}$-agonist amselamine [2-amino-5-(2-aminoethyl)-4-methyl-1,3-selenazole $\quad$ 2. $^{3} \quad \mathrm{~N}$-Acylated 2-amino-4(isothiocyanatomethyl)-1,3-selenazoles (3) and the corresponding 2-carbamates possess antitumoral activity. ${ }^{4}$ Recently, it was reported that 2-piperidino- and 4-phenyl-2-piperidino-1,3-selenazoles exhibit strong superoxide anion-scavening activity ${ }^{5}$, while 2-piperidino- and 2-morpholino-5chloroacetyl-1,3-selenazoles (4a, b) show antioxidant activity and strongly inhibit lipopolysaccharide-induced nitric oxide release from microglial cells. ${ }^{6}$ Moreover, 2-amino-1,3selenazole derivatives found application in the synthesis of organic dyes. ${ }^{7}$

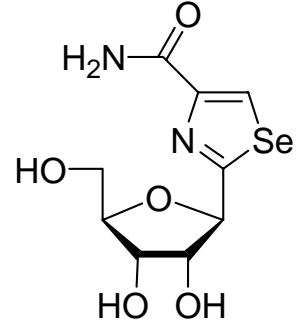

selenazofurin (1)

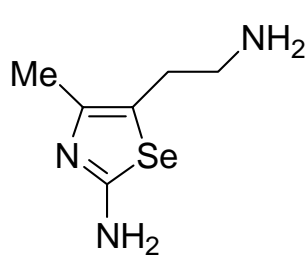

amselamine (2)

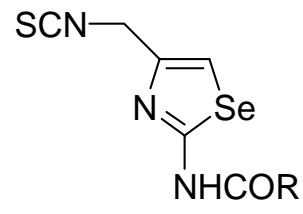

3 R = alkyl, phenyl

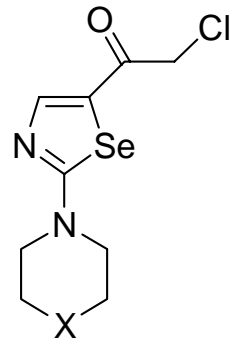

$\mathbf{4 a} \mathrm{X}=\mathrm{CH}_{2} ; \mathbf{b} \mathrm{X}=\mathrm{O}$

Figure 1. Some biologically active 1,3-selenazole derivatives. 
In view of the importance of substituted 1,3-selenazoles, several methods for their preparation have been reported. The 2-amino-1,3-selenazoles are generally prepared via the [3+2] route using the Hantzsch method, which is based on the reaction of selenoureas with $\alpha$-halogenated carbonyl compounds. ${ }^{1}$ The latter starting materials can be easily obtained by chlorination or bromination of aliphatic ketones or other similar carbonyl derivatives. ${ }^{8}$ The reaction of selenourea with $\alpha$ halogenated carbonyl compounds gives a variety of 2-amino-1,3-selenazoles, bearing substituents at 4- and 5-positions of the heterocyclic ring. For example, treatment of selenourea with 1,3dichloroacetone and phenylacetyl bromide afforded 4-chloromethyl- ${ }^{4,9}$ and 4-phenyl-2-amino-1,3selenazole, ${ }^{10}$ respectively, while the reaction with ethyl $\alpha$-bromoacetoacetate gave ethyl 2 -amino-4methyl-1,3-selenazole-5-carboxylate. ${ }^{10,11}$

Recently, we showed that diethyl 2,4-dibromo-3-oxoglutarate can serve as a suitable synthon for the preparation of highly functionalized 2-aminothiazole derivatives. ${ }^{12}$ In an effort to broaden the scope of this reagent in the Hantszch synthesis and to further development of methods for preparation of functionalized 1,3-selenazole derivatives, the objective of this work is to investigate the reaction of diethyl 2,4-dibromo-3-oxoglutarate with selenourea and to study the structure and chemical transformations of the obtained novel cyclic products.

\section{Results and Discussion}

Dibromination of diethyl 3-oxoglutarate 1 with NBS in carbon tetrachloride produced diethyl 2,4dibromo-3-oxoglutarate ${ }^{12} \mathbf{2}$ as a mixture of diastereomers, which was directly used in the next reaction step. The reaction of dibrominated carbonyl compound $\mathbf{2}$ with selenourea was carried out under argon in ethanol at room temperature for $48 \mathrm{~h}$. Then the reaction mixture was treated with a base and the separated material was extracted with an organic solvent. The purification of the crude product by flash chromatography gave ethyl 2-(2-amino-5-ethoxycarbonyl-1,3-selenazol-4-yl)-2bromoethanoate (3) in $61 \%$ yield (Scheme 1).

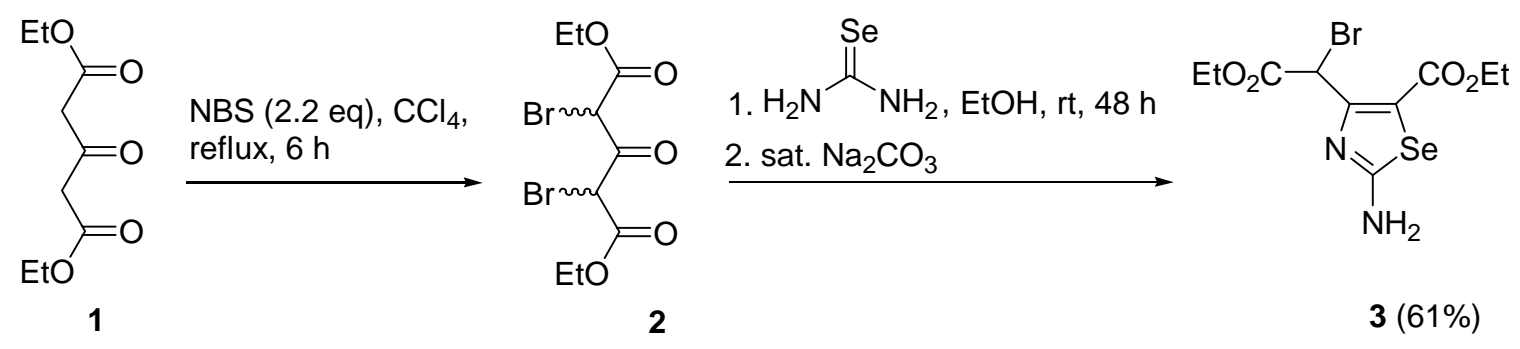

\section{Scheme 1}

The structure assignment of $\mathbf{3}$ was based on spectral data. The IR spectrum shows a broad band at $3151 \mathrm{~cm}^{-1}$ for $\mathrm{NH}_{2}$ and sharp bands at 1737 and $1718 \mathrm{~cm}^{-1}$, which are due to diester $\mathrm{C}=\mathrm{O}$ groups. The ${ }^{1} \mathrm{H}$ NMR spectrum contains the characteristic methine proton $(\mathrm{CHBr})$ signal at $6.52 \mathrm{ppm}$. The 
${ }^{13} \mathrm{C}$ NMR spectrum of $\mathbf{3}$ shows the characteristic signals of the 1,3-selenazole ring skeleton carbons at 113.2, 155.3 and $162.7 \mathrm{ppm}$, while a signal of the methine carbon (CHBr) is situated at $42.2 \mathrm{ppm}$.

The single crystal X-ray structure (Figure 2$)^{13}$ shows that the skeleton of the asymmetric unit contains five-membered 1,3-selenazole ring, with bromo(ethoxycarbonyl)methyl group attached to the atom C(4) and ethoxycarbonyl group attached to the atom C(5). The C(2)-Se and C(5)-Se bond lengths in the molecule 3 are 1.898(4) and 1.881(15) $\AA$, respectively, and they are similar to that found in related structures. ${ }^{6,14}$ The bond angle $\mathrm{C}(2)-\mathrm{Se}-\mathrm{C}(5)$ is $84.3(6)^{\circ}$.

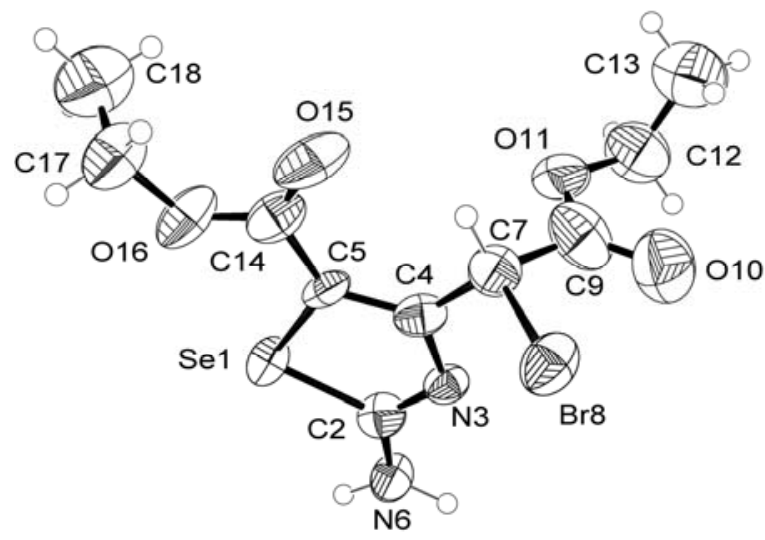

Figure 2. ORTEP drawing of functionalized 2-amino-1,3-selenazole 3.

In order to evaluate the synthetic utility of 2-amino-1,3-selenazole 3 acylation reactions of the amino group have been investigated. 2-Amino-1,3-selenazole 3 was subjected to acetylation with acetic anhydride in the presence of DMAP to give the amide 4a. The IR spectrum of $\mathbf{4 a}$ contains the primary amide bands at $v_{\mathrm{N}-\mathrm{H}}=3159$ and $v_{\mathrm{C}=\mathrm{O}}=1659 \mathrm{~cm}^{-1}$, while the ${ }^{1} \mathrm{H}$ NMR spectrum shows a singlet of the acetyl group protons at $2.20 \mathrm{ppm}$. Treatment of the amine 3 with $\mathrm{Boc}_{2} \mathrm{O}$ in the presence of DMAP gave the $N$-Boc protected product $\mathbf{4 b}$. The ${ }^{1} \mathrm{H}$ NMR spectrum of $\mathbf{4 b}$ contains a singlet of the tert-butoxy group at $1.48 \mathrm{ppm}$. Next, we investigated representative nucleophilic substitution reactions of compound 3 with $\mathrm{KSCN}, \mathrm{NaN}_{3}$, amines and thiols. The nucleophilic displacement of a bromide at the saturated carbon with the thiocyanate group gave a substituted product 5. The IR spectrum of 5 showed a sharp band at $2158 \mathrm{~cm}^{-1}$ assigned to the thiocyanate group. ${ }^{15}$ In its ${ }^{13} \mathrm{C}$ NMR spectrum, the characteristic resonance signal of the thiocyanate carbon appeared at $103.6 \mathrm{ppm}$.

Treatment of $\mathbf{3}$ with sodium azide in DMSO at $60^{\circ} \mathrm{C}$ for $6 \mathrm{~h}$, followed by work-up consisting of dilution with dichloromethane, washing the organic layer with water and concentration under reduced pressure, gave the azido derivative 6 . The absorption band at $2122 \mathrm{~cm}^{-1}$, which is due to the azido group, ${ }^{15}$ is observed in the IR spectrum of $\mathbf{6}$.

Then we turned our attention to study the possibility of substitution of a bromide by an amino group. The reaction of the bromide $\mathbf{3}$ with methyl amine hydrochloride in ethanol in the presence of bases, led to the formation of the secondary amine 7a. The IR spectra of 7a showed broad bands in the area $3324-3180 \mathrm{~cm}^{-1}$ for the $\mathrm{NH}_{2}$ and $\mathrm{NH}$ groups and the intense bands at 1738 and $1696 \mathrm{~cm}^{-1}$ 
assigned to the carbonyl groups of the diester. When compound $\mathbf{3}$ was reacted with benzylamine, the secondary amine $7 \mathbf{b}$ was obtained in $62 \%$ yield.
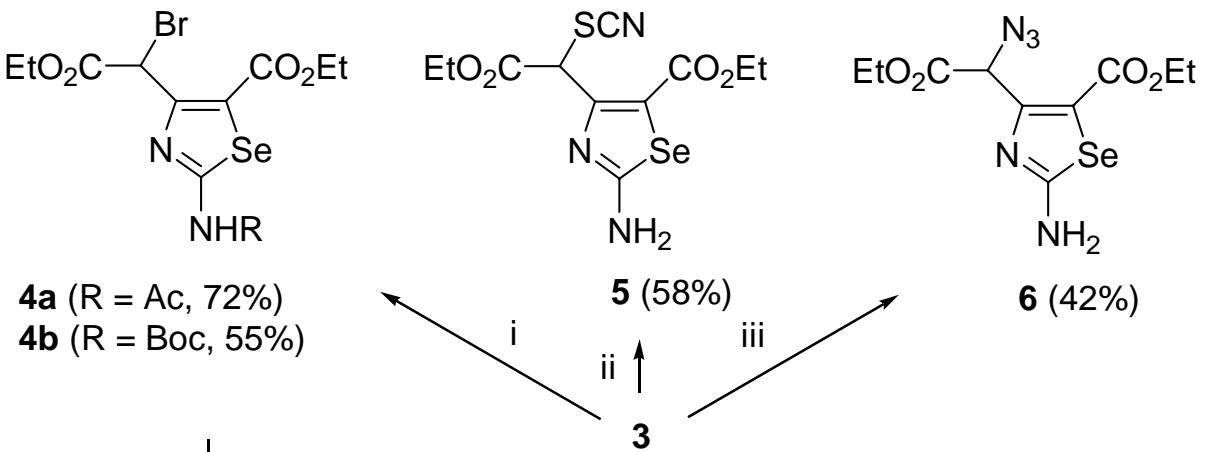

$6(42 \%)$
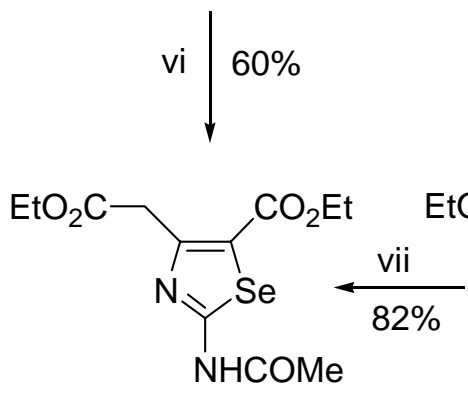

9

$8(60 \%)$

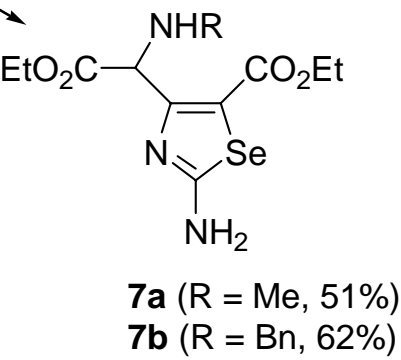

Scheme 2. Reagents and conditions: $\mathrm{i}, \mathrm{Ac}_{2} \mathrm{O}$, DMAP, DCM, reflux, $6 \mathrm{~h}$, or $\mathrm{Boc}_{2} \mathrm{O}$, DMAP, DCM, reflux, 4 h; ii, KSCN, acetone, rt, 48 h; iii, $\mathrm{NaN}_{3}$, DMSO, $60{ }^{\circ} \mathrm{C}, 6 \mathrm{~h}$; iv, $\mathrm{MeNH}_{2} \cdot \mathrm{HCl}$ or $\mathrm{BnNH}_{2}$, $\mathrm{K}_{2} \mathrm{CO}_{3}$, TEA, EtOH, rt, 5 days; v, EtSH, TEA, EtOH, rt, 5 days; vi, EtSH, TEA, EtOH, rt, 5 days; vii, $\mathrm{Ac}_{2} \mathrm{O}$, DMAP, DCM, reflux, $6 \mathrm{~h}$.

To assess further its reactivity, compound $\mathbf{3}$ was treated with thiols. However, the reaction of the bromide 3 with ethanethiol in the presence of TEA did not give the desired sulfide, instead debrominated compound $\mathbf{8}$ was obtained. The reaction with methyl thioglycolate in similar conditions afforded the same product 8 . The ${ }^{1} \mathrm{H}$ NMR spectrum of $\mathbf{8}$ revealed a singlet at $3.85 \mathrm{ppm}$ for methylene protons $\left(\mathrm{CH}_{2} \mathrm{CO}\right) .{ }^{13} \mathrm{C}$ NMR spectrum showed the presence of three methylene carbons at $36.6\left(\mathrm{CH}_{2} \mathrm{CO}\right), 60.0\left(\mathrm{OCH}_{2}\right), 60.1\left(\mathrm{OCH}_{2}\right)$, respectively. Similarly, treatment of 2acetylamino-1,3-selenazole $\mathbf{4 a}$ with ethanethiol afforded the corresponding debrominated compound 9. The latter compound was also obtained by reaction of $\mathbf{8}$ with acetic anhydride in the presence of DMAP.

The use of thiols as catalysts and hydrogen donors for radical chain reduction of organic halides is well documented in the literature. ${ }^{16}$ For example, 1,2,2,6,6-pentamethylpiperidine/ mercaptoethanol system was shown to be an effective reducing agent for the radical chain reduction of bromoesters. ${ }^{16 \mathrm{c}}$ Therefore, it can be assumed, that ethanethiol/TEA or methyl thioglycolate/TEA systems possess similar reductive properties and accomplish dehalogenation of bromoesters $\mathbf{3}$ and $\mathbf{4 a}$ by a radical-chain mechanism. 


\section{Conclusions}

In summary, an efficient route for the preparation of a wide variety of highly functionalized 1,3selenazoles has been developed. This method allows functionalized 1,3-selenazoles to be easily available for further investigations into their chemical and biological properties.

\section{Experimental Section}

General. Melting points were determined in open capillary tubes with a Büchi B-540 melting point apparatus and are uncorrected. Infrared spectra were recorded on a Perkin Elmer Spectrum One spectrometer using potassium bromide pellets. ${ }^{1} \mathrm{H}$ NMR spectra were recorded at $300 \mathrm{MHz}$ on a Varian Unity Inova spectrometer. ${ }^{13} \mathrm{C}$ NMR spectra were registered at $75 \mathrm{MHz}$ using the instrument mentioned above. Chemical shifts, expressed in ppm, were relative to tetramethylsilane (TMS). Mass spectra were measured using Waters ZQ 2000 instrument (ion spray). Diffraction data were collected on Bruker-Nonius KappaCCD diffractometer at room temperature and also at $-100{ }^{\circ} \mathrm{C}$. The crystal structures were solved using known programs. ${ }^{17}$ Elemental analyses were performed by the Microanalytical Laboratory, Department of Organic Chemistry, Kaunas University of Technology, Lithuania. For thin layer chromatographic (TLC) analyses, Merck precoated TLC plates (silica gel 60 F254) were used.

Ethyl 2-(2-amino-5-ethoxycarbonyl-1,3-selenazol-4-yl)-2-bromoethanoate (3). To a solution of selenourea $(1.60 \mathrm{~g}, 13 \mathrm{mmol})$ in absolute ethanol $(50 \mathrm{~mL})$ 2,4-dibromo-3-oxoglutarate $(4.68 \mathrm{~g}, 13$ mmol) was added dropwise and the mixture was stirred under argon at $\mathrm{rt}$ for $48 \mathrm{~h}$. The solvent was removed at reduced pressure and the residue was dissolved in $100 \mathrm{~mL}$ of water. The $\mathrm{pH}$ was then adjusted to $\mathrm{pH} 9$ with sat. $\mathrm{Na}_{2} \mathrm{CO}_{3}$ and the aqueous solution was extracted with diethyl ether $(3 \times 50$ $\mathrm{mL}$ ). The combined organic extracts were washed with brine and dried over anhydrous $\mathrm{Na}_{2} \mathrm{SO}_{4}$. The solvent was removed under reduced pressure and the residue was subjected to flash chromatography on silica gel (hexane - ethyl acetate, $4: 1$ ) to afford the title compound $\mathbf{3}$ as pale yellow crystals (3.05 g, 61\%), mp 179-180 ${ }^{\circ} \mathrm{C}$ (from ethanol). ${ }^{1} \mathrm{H}$ NMR (300 MHz, DMSO-d $\mathrm{d}_{6}$ ): $\delta$ (ppm) $1.15\left(3 \mathrm{H}, \mathrm{t}, J=7.1 \mathrm{~Hz}, \mathrm{CH}_{3}\right), 1.23\left(3 \mathrm{H}, \mathrm{t}, J=7.1 \mathrm{~Hz}, \mathrm{CH}_{3}\right), 4.14\left(2 \mathrm{H}, \mathrm{q}, J=7.1 \mathrm{~Hz}, \mathrm{CH}_{2}\right)$, $4.19\left(2 \mathrm{H}, \mathrm{q}, J=7.1 \mathrm{~Hz}, \mathrm{CH}_{2}\right), 6.52(1 \mathrm{H}, \mathrm{s}, \mathrm{CH}), 8.32\left(2 \mathrm{H}, \mathrm{br} \mathrm{s}, \mathrm{NH}_{2}\right) .{ }^{13} \mathrm{C}$ NMR $(75 \mathrm{MHz}$, DMSO$\left.\mathrm{d}_{6}\right): \delta(\mathrm{ppm}) 13.9\left(\mathrm{CH}_{3}\right), 14.2\left(\mathrm{CH}_{3}\right), 42.2(\mathrm{CH}), 60.7\left(\mathrm{CH}_{2}\right), 62.2\left(\mathrm{CH}_{2}\right)$ 113.2 (C-5), $155.3(\mathrm{C}-4)$, $162.7(\mathrm{C}-2), 165.6(\mathrm{C}=\mathrm{O}), 172.4(\mathrm{C}=\mathrm{O})$. MS $m / z(\%)$ : 383/85 $\left(\mathrm{M}^{+}, 42 / 74\right)$. Anal. Calcd for $\mathrm{C}_{10} \mathrm{H}_{13} \mathrm{BrN}_{2} \mathrm{O}_{4} \mathrm{Se}: \mathrm{C} 31.27 ; \mathrm{H} 3.41 ; \mathrm{N}$ 7.29. Found: $\mathrm{C} 31.67 ; \mathrm{H} 3.53 ; \mathrm{N} 7.17$.

Ethyl 2-(2-acetylamino-5-ethoxycarbonyl-1,3-selenazol-4-yl)-2-bromoethanoate (4a). To a precooled in an ice bath solution of 2-amino-1,3-selenazole $3(300 \mathrm{mg}, 0.78 \mathrm{mmol})$ ) and DMAP $(125 \mathrm{mg}, 1.02 \mathrm{mmol})$ in dry dichloromethane $(8 \mathrm{~mL})$ a solution of acetic anhydride $(150 \mathrm{mg}, 0.14$ $\mathrm{mL}, 1.5 \mathrm{mmol})$ in dichloromethane $(2 \mathrm{~mL})$ was added dropwise. The reaction mixture was allowed to reach $\mathrm{rt}$ and stirred under argon for $6 \mathrm{~h}$, then washed with brine and dried over anhydrous $\mathrm{Na}_{2} \mathrm{SO}_{4}$. The solvent was removed under reduced pressure and the residue material was subjected to 
flash chromatography with silica gel using a solvent gradient (from 20 to $50 \%$ ethyl acetate in hexane) to give the title compound $4 \mathbf{a}$ as white crystals $\left(240 \mathrm{mg}, 72 \%\right.$ ), mp $120-121{ }^{\circ} \mathrm{C}$ (from ethyl acetate - hexane). ${ }^{1} \mathrm{H}$ NMR (300 MHz, DMSO-d 6 ): $\delta(\mathrm{ppm}) 1.14\left(3 \mathrm{H}, \mathrm{t}, J=7.1 \mathrm{~Hz}, \mathrm{CH}_{2} \mathrm{CH}_{3}\right), 1.29$ $\left(3 \mathrm{H}, \mathrm{t}, J=7.1 \mathrm{~Hz}, \mathrm{CH}_{2} \mathrm{CH}_{3}\right), 2.20\left(3 \mathrm{H}, \mathrm{s}, \mathrm{COCH}_{3}\right), 4.16\left(2 \mathrm{H}, \mathrm{q}, J=7.1 \mathrm{~Hz}, \mathrm{CH}_{2}\right), 4.27(2 \mathrm{H}, \mathrm{q}, J=$ 7.1, $\left.\mathrm{CH}_{2}\right), 6.66(1 \mathrm{H}, \mathrm{s}, \mathrm{CH}), 13.12(1 \mathrm{H}, \mathrm{br} \mathrm{s}, \mathrm{NH}) .{ }^{13} \mathrm{C} \mathrm{NMR}\left(75 \mathrm{MHz}, \mathrm{DMSO}-\mathrm{d}_{6}\right): \delta(\mathrm{ppm}) 13.9$ $\left(\mathrm{CH}_{2} \mathrm{CH}_{3}\right), 14.0\left(\mathrm{CH}_{2} \mathrm{CH}_{3}\right), 22.2\left(\mathrm{COCH}_{3}\right), 42.2(\mathrm{CH}), 61.3\left(\mathrm{CH}_{2}\right), 62.4\left(\mathrm{CH}_{2}\right), 120.8(\mathrm{C}-5), 152.6$ $(\mathrm{C}-4), 162.5,162.9(\mathrm{C}-2$ and $\mathrm{C}=\mathrm{O}), 165.7(\mathrm{C}=\mathrm{O}), 170.3(\mathrm{C}=\mathrm{O})$. IR $\left(\mathrm{KBr}, \mathrm{cm}^{-1}\right): v_{\mathrm{N}-\mathrm{H}}=3159 ; v_{\mathrm{C}=\mathrm{O}}=$ $1762 ; v_{\mathrm{C}=\mathrm{O}}=1697 ; v_{\mathrm{C}=\mathrm{O}}=1659$. Anal. Calcd for $\mathrm{C}_{12} \mathrm{H}_{15} \mathrm{BrN}_{2} \mathrm{O}_{5} \mathrm{Se}$ : C 33.82; H 3.55; N 6.57. Found: C $34.11 ; \mathrm{H} 3.74 ; \mathrm{N} 6.33$.

Ethyl 2-(2-tert-butoxycarbonylamino-5-ethoxycarbonyl-1,3-selenazol-4-yl)-2-bromoethanoate (4b) was obtained similarly to $4 \mathbf{a}$ from $3(300 \mathrm{mg}, 0.78 \mathrm{mmol})$ and $\mathrm{Boc}_{2} \mathrm{O}(200 \mathrm{mg}, 0.94 \mathrm{mmol})$ in $210 \mathrm{mg}$ yield (55\%) with mp $124-125{ }^{\circ} \mathrm{C}$ (ethyl acetate - hexane). ${ }^{1} \mathrm{H}$ NMR (300 MHz, DMSO-d 6 ): $\delta(\mathrm{ppm}) 1.14\left(3 \mathrm{H}, \mathrm{t}, J=7.1, \mathrm{CH}_{2} \mathrm{CH}_{3}\right), 1.28\left(3 \mathrm{H}, \mathrm{t}, J=7.1, \mathrm{CH}_{2} \mathrm{CH}_{3}\right), 1.48\left(9 \mathrm{H}, \mathrm{s}, 3 \mathrm{xCH}_{3}\right), 4.13$ $\left(2 \mathrm{H}, \mathrm{q}, J=7.1, \mathrm{CH}_{2}\right), 4.28\left(2 \mathrm{H}, \mathrm{q}, J=7.1, \mathrm{CH}_{2}\right), 6.62(1 \mathrm{H}, \mathrm{s}, \mathrm{CH}), 12.50(1 \mathrm{H}, \mathrm{br} \mathrm{s}, \mathrm{NH}) .{ }^{13} \mathrm{C}$ NMR (75 MHz, DMSO-d 6$): \delta(\mathrm{ppm}) 13.8\left(\mathrm{CH}_{2} \mathrm{CH}_{3}\right), 13.9\left(\mathrm{CH}_{2} \mathrm{CH}_{3}\right), 27.7\left[3 \mathrm{C}, \mathrm{C}\left(\mathrm{CH}_{3}\right)_{3}\right], 42.2(\mathrm{CH})$, $61.3\left(\mathrm{CH}_{2}\right), 62.4\left(\mathrm{CH}_{2}\right), 82.6\left[C\left(\mathrm{CH}_{3}\right)_{3}\right], 120.6(\mathrm{C}-5), 153.2,153.5,162.7,165.0,165.5 . \mathrm{IR}(\mathrm{KBr}$, $\left.\mathrm{cm}^{-1}\right): v_{\mathrm{N}-\mathrm{H}}=3256 ; v_{\mathrm{C}=\mathrm{O}}=1762 ; v_{\mathrm{C}=\mathrm{O}}=1720 ; v_{\mathrm{C}=\mathrm{O}}=1679 . \mathrm{MS} m / z(\%): 507 / 509\left(\mathrm{M}+\mathrm{H}^{+}, \mathrm{Na}\right.$; 52/42). Anal. Calcd for $\mathrm{C}_{15} \mathrm{H}_{21} \mathrm{BrN}_{2} \mathrm{O}_{6}$ Se: C 37.21; H 4.37; N 5.79. Found: C 37.46; H 4.27; N 5.91.

Ethyl 2-(2-amino-5-ethoxycarbonyl-1,3-selenazol-4-yl)-2-thiocyanatoethanoate (5). To a solution of 2-amino-1,3-selenazole $3(300 \mathrm{mg}, 0.78 \mathrm{mmol})$ in dry acetone $(10 \mathrm{~mL}) \mathrm{KSCN}(91 \mathrm{mg}$, $0.94 \mathrm{mmol}$ ) was added and the reaction mixture was stirred under argon at $\mathrm{rt}$ for $48 \mathrm{~h}$. Then it was concentrated under reduced pressure. The resulting residue was diluted with dichloromethane (15 $\mathrm{mL}$ ) and the solid was removed by filtration. The solvent was evaporated under reduced pressure and the residue subjected to flash chromatography with silica gel (hexane - ethyl acetate, $3: 1$ ) to give the title compound 5 as white crystals $\left(110 \mathrm{mg}, 58 \%\right.$ ), mp 152-153 ${ }^{\circ} \mathrm{C}$ (from ethyl acetate hexane). ${ }^{1} \mathrm{H}$ NMR $\left(300 \mathrm{MHz}, \mathrm{DMSO}_{6}\right): \delta(\mathrm{ppm}) 1.16\left(3 \mathrm{H}, \mathrm{t}, J=7.2 \mathrm{~Hz}, \mathrm{CH}_{3}\right), 1.23(3 \mathrm{H}, \mathrm{t}, J=$ 7.2, $\left.\mathrm{CH}_{3}\right), 4.16\left(2 \mathrm{H}, \mathrm{q}, J=7.2 \mathrm{~Hz}, \mathrm{CH}_{2}\right), 4.17\left(2 \mathrm{H}, \mathrm{q}, J=7.2 \mathrm{~Hz}, \mathrm{CH}_{2}\right), 6.12(1 \mathrm{H}, \mathrm{s}, \mathrm{CH}), 8.16(2 \mathrm{H}$, br s, $\left.\mathrm{NH}_{2}\right) .{ }^{13} \mathrm{C}$ NMR (75 MHz, DMSO-d $): \delta(\mathrm{ppm}) 13.8\left(\mathrm{CH}_{3}\right), 14.1\left(\mathrm{CH}_{3}\right), 43.7(\mathrm{CH}), 60.6\left(\mathrm{CH}_{2}\right)$, $62.2\left(\mathrm{CH}_{2}\right), 103.6(\mathrm{SCN}), 109.8(\mathrm{C}-5), 152.2(\mathrm{C}-4), 161.0,168.0(\mathrm{C}-2$ and $\mathrm{C}=\mathrm{O}), 170.4(\mathrm{C}=\mathrm{O})$. IR $\left(\mathrm{KBr}, \mathrm{cm}^{-1}\right): v_{\mathrm{N}-\mathrm{H}}=3389 ; v_{\mathrm{N}-\mathrm{H}}=3282 ; v_{\mathrm{SCN}}=2158 ; v_{\mathrm{C}=\mathrm{O}}=1721 ; v_{\mathrm{C}=\mathrm{O}}=1691 . \mathrm{MS} \mathrm{m} / \mathrm{z}(\%): 362$ $\left(\mathrm{M}^{+}, 12\right)$. Anal. Calcd for $\mathrm{C}_{11} \mathrm{H}_{13} \mathrm{~N}_{3} \mathrm{O}_{4} \mathrm{SSe}$ : C 36.47; H 3.62; N 11.60. Found: $\mathrm{C} 36.68 ; \mathrm{H} \mathrm{4.01;} \mathrm{N}$ 11.55 .

Ethyl 2-(2-amino-5-ethoxycarbonyl-1,3-selenazol-4-yl)-2-azidoethanoate (6). To a solution of 2amino-1,3-selenazole $3(0.5 \mathrm{~g}, 1.30 \mathrm{mmol})$ in dry DMSO (8 mL) sodium azide (90 $\mathrm{mg}, 1.40 \mathrm{mmol})$ was added. The mixture was heated with stirring under argon at $60{ }^{\circ} \mathrm{C}$ for $6 \mathrm{~h}$, then the resulting solution was allowed to reach $\mathrm{rt}$ and diluted with dichloromethane $(50 \mathrm{~mL})$. The organic solution was washed with brine $(3 \times 20 \mathrm{~mL})$ and dried over anhydrous $\mathrm{Na}_{2} \mathrm{SO}_{4}$. The solvent was removed under reduced pressure and the residue material was subjected to flash chromatography with silica gel using a solvent gradient (from 25 to $50 \%$ ethyl acetate in hexane) to give the title compound 6 as white crystals (190 mg, 42\%), mp 163-164 ${ }^{\circ} \mathrm{C}$ (dichloromethane). ${ }^{1} \mathrm{H}$ NMR (300 MHz, DMSO-d 6 ): $\delta(\mathrm{ppm}) 1.15\left(3 \mathrm{H}, \mathrm{t}, J=7.2 \mathrm{~Hz}, \mathrm{CH}_{3}\right), 1.22\left(3 \mathrm{H}, \mathrm{t}, J=7.2 \mathrm{~Hz}, \mathrm{CH}_{3}\right), 4.11-4.21\left(4 \mathrm{H}, \mathrm{m}, 2 \mathrm{xCH}_{2}\right)$, 
$5.76(1 \mathrm{H}, \mathrm{s}, \mathrm{CH}), 8.33\left(2 \mathrm{H}, \mathrm{br} \mathrm{s}, \mathrm{NH}_{2}\right) .{ }^{13} \mathrm{C}$ NMR $\left(75 \mathrm{MHz}, \mathrm{DMSO}-\mathrm{d}_{6}\right): \delta(\mathrm{ppm}) 13.9\left(\mathrm{CH}_{3}\right), 14.1$ $\left(\mathrm{CH}_{3}\right), 57.5(\mathrm{CH}), 60.6\left(\mathrm{CH}_{2}\right), 61.6\left(\mathrm{CH}_{2}\right), 113.8(\mathrm{C}-5), 154.1(\mathrm{C}-4), 162.8,167.6(\mathrm{C}-2$ and $\mathrm{C}=\mathrm{O})$, $173.1(\mathrm{C}=\mathrm{O})$. IR $\left(\mathrm{KBr}, \mathrm{cm}^{-1}\right): v_{\mathrm{N}-\mathrm{H}}=3407 ; v_{\mathrm{N}-\mathrm{H}}=3290 ; v_{\text {azide }}=2122 ; v_{\mathrm{C}=\mathrm{O}}=1744 ; v_{\mathrm{C}=\mathrm{O}}=1696$. MS $\mathrm{m} / \mathrm{z}(\%): 370\left(\mathrm{M}+\mathrm{H}^{+}, \mathrm{Na} ; 58\right)$. Anal. Calcd for $\mathrm{C}_{10} \mathrm{H}_{13} \mathrm{~N}_{5} \mathrm{O}_{4} \mathrm{Se}$ : C 34.69; H 3.78\%; N 20.23. Found: C 34.75; H 3.98; N 19.90 .

Ethyl 2-(2-amino-5-ethoxycarbonyl-1,3-selenazol-4-yl)-2-methylaminoethanoate (7a). To a solution of 2-amino-1,3-selenazole $3(0.3 \mathrm{~g}, 0.78 \mathrm{mmol})$ in absolute ethanol $(8 \mathrm{~mL}) \mathrm{K}_{2} \mathrm{CO}_{3}(0.14 \mathrm{~g}$, $1.0 \mathrm{mmol})$, triethylamine $(0.2 \mathrm{~g}, 0.25 \mathrm{~mL}, 2.0 \mathrm{mmol})$ and methylamine hydrochloride $(0.1 \mathrm{~g}, 1.48$ mmol) were added. The mixture was stirred under argon at rt for 5 days. The solvent was removed under reduced pressure, water $(15 \mathrm{~mL})$ was added to the residue and the mixture was extracted with dichloromethane $(3 \times 20 \mathrm{~mL})$. The combined organic extracts were washed with brine and dried over anhydrous $\mathrm{Na}_{2} \mathrm{SO}_{4}$. The solvent was removed under reduced pressure and the residue was twice recrystallized from dichloromethane to afford the title compound $\mathbf{7 a}$ as white crystals (133 $\mathrm{mg}, 51 \%), \mathrm{mp} 176-17{ }^{\circ} \mathrm{C} .{ }^{1} \mathrm{H}$ NMR (300 MHz, DMSO-d 6 ): $\delta(\mathrm{ppm}) 1.11(3 \mathrm{H}, \mathrm{t}, J=7.2 \mathrm{~Hz}$, $\left.\mathrm{CH}_{2} \mathrm{CH}_{3}\right), 1.22\left(3 \mathrm{H}, \mathrm{t}, J=7.2 \mathrm{~Hz}, \mathrm{CH}_{2} \mathrm{CH}_{3}\right), 2.21\left(3 \mathrm{H}, \mathrm{s}, \mathrm{NCH}_{3}\right), 4.05\left(2 \mathrm{H}, \mathrm{q}, J=7.2 \mathrm{~Hz}, \mathrm{CH}_{2} \mathrm{CH}_{3}\right)$, $4.14\left(2 \mathrm{H}, \mathrm{q}, J=7.2 \mathrm{~Hz}, \mathrm{CH}_{2} \mathrm{CH}_{3}\right), 5.15(1 \mathrm{H}, \mathrm{s}, \mathrm{CH}), 8.21\left(2 \mathrm{H}, \mathrm{br} \mathrm{s}, \mathrm{NH}_{2}\right) .{ }^{13} \mathrm{C} \mathrm{NMR}(75 \mathrm{MHz}$, DMSO-d $\left.{ }_{6}\right): \delta(\mathrm{ppm}) 14.1\left(\mathrm{CH}_{2} \mathrm{CH}_{3}\right), 14.2\left(\mathrm{CH}_{2} \mathrm{CH}_{3}\right), 33.56\left(\mathrm{NCH}_{3}\right), 45.30(\mathrm{CH}), 60.2\left(\mathrm{CH}_{2}\right), 60.3$ $\left(\mathrm{CH}_{2}\right), 112.8(\mathrm{C}-5), 158.8(\mathrm{C}-4), 163.1,170.5(\mathrm{C}-2$ and $\mathrm{C}=\mathrm{O}), 172.8(\mathrm{C}=\mathrm{O}) . \mathrm{IR}\left(\mathrm{KBr}, \mathrm{cm}^{-1}\right): v_{\mathrm{N}-\mathrm{H}}=$ 3324; $v_{\mathrm{N}-\mathrm{H}}=3180 ; v_{\mathrm{C}=\mathrm{O}}=1738 ; v_{\mathrm{C}=\mathrm{O}}=1696$. MS m/z (\%): $334\left(\mathrm{M}^{+}, 20\right), 358\left(\mathrm{M}+\mathrm{H}^{+}, \mathrm{Na} ; 36\right)$. Anal. Calcd for $\mathrm{C}_{11} \mathrm{H}_{17} \mathrm{~N}_{3} \mathrm{O}_{4}$ Se: $\mathrm{C} 39.53 ; \mathrm{H} 5.13$; N 12.57. Found: C 39.40; H 5.19; N 12.11.

Ethyl 2-(2-amino-5-ethoxycarbonyl-1,3-selenazol-4-yl)-2-benzylaminoethanoate (7b) was obtained similarly to $7 \mathbf{a}$ from $3(0.3 \mathrm{~g}, 0.78 \mathrm{mmol})$ and benzylamine $(167 \mathrm{mg}, 0.17 \mathrm{ml}, 1.56 \mathrm{mmol})$ in $198 \mathrm{mg}$ yield (62\%) with mp 168-169 ${ }^{\circ} \mathrm{C}$ (dichloromethane). ${ }^{1} \mathrm{H}$ NMR (300 MHz, DMSO-d 6 ): $\delta$ (ppm) $1.11\left(3 \mathrm{H}, \mathrm{t}, J=6.9 \mathrm{~Hz}, \mathrm{CH}_{3}\right), 1.15\left(3 \mathrm{H}, \mathrm{t}, J=6.9 \mathrm{~Hz}, \mathrm{CH}_{3}\right), 2.45(1 \mathrm{H}$, br s, NH), $3.63(2 \mathrm{H}, \mathrm{s}$, $\left.\mathrm{NCH}_{2}\right), 4.00-4.15\left(4 \mathrm{H}, \mathrm{m}, 2 \mathrm{xOCH}_{2}\right), 5.27(1 \mathrm{H}, \mathrm{s}, \mathrm{CH}), 7.28-7.30(5 \mathrm{H}, \mathrm{m}, \mathrm{ArH}), 8.17(2 \mathrm{H}$, br s, $\left.\mathrm{NH}_{2}\right) .{ }^{13} \mathrm{C}$ NMR $\left(75 \mathrm{MHz}, \mathrm{DMSO}-\mathrm{d}_{6}\right): \delta(\mathrm{ppm}) 14.0\left(\mathrm{CH}_{3}\right), 14.1\left(\mathrm{CH}_{3}\right), 50.0,58.0,60.1,60.2$, $113.0,126.7,127.8(2 \mathrm{C}), 128.1$ (2C), 140.0, 158.7, 162.9, 170.6, 172.6. IR $\left(\mathrm{KBr}, \mathrm{cm}^{-1}\right): v_{\mathrm{N}-\mathrm{H}}=$ $3303 ; v_{\mathrm{N}-\mathrm{H}}=3104 ; v_{\mathrm{C}=\mathrm{O}}=1731 ; v_{\mathrm{C}=\mathrm{O}}=1689 . \mathrm{MS} \mathrm{m} / \mathrm{z}(\%): 434\left(\mathrm{M}+\mathrm{H}^{+}, \mathrm{Na} ; 68\right)$. Anal. Calcd for $\mathrm{C}_{17} \mathrm{H}_{21} \mathrm{~N}_{3} \mathrm{O}_{4} \mathrm{Se}: \mathrm{C} 49.76$; H 5.16; N 10.24. Found: C 49.39; H 5.19; N 9.98.

Ethyl 2-(2-amino-5-ethoxycarbonyl-1,3-selenazol-4-yl)ethanoate (8). To solution of 2-amino1,3-selenazole $3(0.3 \mathrm{~g}, 0.78 \mathrm{mmol})$ and triethyl amine $(0.15 \mathrm{~g}, 0.2 \mathrm{~mL}, 1.48 \mathrm{mmol})$ in absolute ethanol $(8 \mathrm{~mL})$ ethanethiol $(0.24 \mathrm{~g}, 0.28 \mathrm{~mL}, 3.9 \mathrm{mmol})$ was added dropwise and the reaction mixture was stirred under argon at $\mathrm{rt}$ for 5 days. The solvent was evaporated under reduced pressure, the resulting residue was diluted with dichloromethane $(10 \mathrm{~mL})$ and the solid was removed by filtration. The organic extract was washed with brine and dried over anhydrous $\mathrm{Na}_{2} \mathrm{SO}_{4}$. The solvent was removed under reduced pressure and the residue material was subjected to flash chromatography with silica gel using a solvent gradient (from 20 to $50 \%$ ethyl acetate in hexane) to give the title compound 8 as white crystals (144 mg, 60\%), mp 124-125 ${ }^{\circ} \mathrm{C}$ (ethyl acetate - hexane). ${ }^{1} \mathrm{H}$ NMR (300 MHz, DMSO-d 6 ): $\delta(\mathrm{ppm}) 1.17\left(3 \mathrm{H}, \mathrm{t}, J=7.2 \mathrm{~Hz}, \mathrm{CH}_{3}\right), 1.20(3 \mathrm{H}, \mathrm{t}, J=$ $\left.7.2 \mathrm{~Hz}, \mathrm{CH}_{3}\right), 3.85\left(2 \mathrm{H}, \mathrm{s}, \mathrm{CH}_{2} \mathrm{CO}\right), 4.05\left(2 \mathrm{H}, \mathrm{q}, J=7.2 \mathrm{~Hz}, \mathrm{CH}_{2} \mathrm{CH}_{3}\right), 4.10(2 \mathrm{H}, \mathrm{q}, J=7.2 \mathrm{~Hz}$, $\left.\mathrm{CH}_{2} \mathrm{CH}_{3}\right), 8.10\left(2 \mathrm{H}\right.$, br s, $\left.\mathrm{NH}_{2}\right) .{ }^{13} \mathrm{C} \mathrm{NMR}\left(75 \mathrm{MHz}, \mathrm{DMSO}-\mathrm{d}_{6}\right): \delta(\mathrm{ppm}) 14.03\left(\mathrm{CH}_{3}\right), 14.20\left(\mathrm{CH}_{3}\right)$, 
$36.6\left(\mathrm{CH}_{2} \mathrm{CO}\right), 60.0\left(\mathrm{OCH}_{2}\right), 60.1\left(\mathrm{OCH}_{2}\right), 112.3(\mathrm{C}-5), 155.7(\mathrm{C}-4), 163.2,169.2(\mathrm{C}-2$ and $\mathrm{C}=\mathrm{O})$, $172.1(\mathrm{C}=\mathrm{O})$. IR $\left(\mathrm{KBr}, \mathrm{cm}^{-1}\right): v_{\mathrm{N}-\mathrm{H}}=3351 ; v_{\mathrm{N}-\mathrm{H}}=3302 ; v_{\mathrm{C}=\mathrm{O}}=1716 ; v_{\mathrm{C}=\mathrm{O}}=1691 . \mathrm{MS} \mathrm{m} / \mathrm{z}(\%): 329$ $\left(\mathrm{M}+\mathrm{H}^{+}, \mathrm{Na} ; 50\right)$. Anal. Calcd for $\mathrm{C}_{10} \mathrm{H}_{14} \mathrm{~N}_{2} \mathrm{O}_{4} \mathrm{Se}: 39.36 ; \mathrm{H} 4.62 ; \mathrm{N}$ 9.18. Found: $39.11 ; \mathrm{H} 4.68 ; \mathrm{N}$ 9.55 .

Ethyl 2-(2-acetylamino-5-ethoxycarbonyl-1,3-selenazol-4-yl)ethanoate (9). Method A. To a solution of 2-acetamino-1,3-selenazole $4 \mathbf{a}(0.3 \mathrm{~g}, 0.86 \mathrm{mmol})$ in absolute ethanol $(8 \mathrm{~mL})$ triethylamine $(0.15 \mathrm{~g}, 0.2 \mathrm{~mL}, 1.48 \mathrm{mmol})$ and ethanethiol $(0.24 \mathrm{~g}, 0.28 \mathrm{~mL}, 3.9 \mathrm{mmol})$ were added. The reaction mixture was stirred under argon at $\mathrm{rt}$ for 5 days. The solvent was evaporated under reduced pressure, the resulting residue was diluted with dichloromethane $(15 \mathrm{~mL})$ and solid was removed by filtration. The extract was washed with $1 \mathrm{M}$ sulphuric acid $(15 \mathrm{~mL})$, brine $(15 \mathrm{~mL})$ and dried over anhydrous $\mathrm{Na}_{2} \mathrm{SO}_{4}$. The solvent was removed under reduced pressure and the residue material was subjected to flash chromatography with silica gel using a solvent gradient (from 20 to $50 \%$ ethyl acetate in hexane) to give the title compound 9 as white crystals $(0.142 \mathrm{~g}, 60 \%)$, mp 141$142{ }^{\circ} \mathrm{C}$ (ethyl acetate - hexane). ${ }^{1} \mathrm{H}$ NMR $\left(300 \mathrm{MHz}, \mathrm{DMSO}-\mathrm{d}_{6}\right): \delta(\mathrm{ppm}) 1.17(3 \mathrm{H}, \mathrm{t}, J=7.2 \mathrm{~Hz}$, $\left.\mathrm{CH}_{2} \mathrm{CH}_{3}\right), 1.26\left(3 \mathrm{H}, \mathrm{t}, J=7.2 \mathrm{~Hz}, \mathrm{CH}_{2} \mathrm{CH}_{3}\right), 2.19\left(3 \mathrm{H}, \mathrm{s}, \mathrm{CH}_{3} \mathrm{CO}\right), 4.04\left(2 \mathrm{H}, \mathrm{s}, \mathrm{CH}_{2} \mathrm{CO}\right), 4.08(2 \mathrm{H}$, q, $\left.J=7.2 \mathrm{~Hz}, \mathrm{CH}_{2} \mathrm{CH}_{3}\right), 4.20\left(2 \mathrm{H}, \mathrm{q}, J=7.2 \mathrm{~Hz}, \mathrm{CH}_{2} \mathrm{CH}_{3}\right), 12.83(1 \mathrm{H}, \mathrm{br} \mathrm{s}, \mathrm{NH}) .{ }^{13} \mathrm{C}$ NMR $(75$ MHz, DMSO-d 6$): \delta(\mathrm{ppm}) 14.0\left(2 \mathrm{xCH}_{3}\right), 22.4\left(\mathrm{CH}_{3}\right), 36.8\left(\mathrm{CH}_{2} \mathrm{CO}\right), 60.3\left(\mathrm{OCH}_{2}\right), 60.6\left(\mathrm{OCH}_{2}\right)$, $120.0(\mathrm{C}-5), 152.5(\mathrm{C}-4), 161.8,163.5,169.1,169.8 . \mathrm{IR}\left(\mathrm{KBr}, \mathrm{cm}^{-1}\right): v_{\mathrm{N}-\mathrm{H}}=3227 ; v_{\mathrm{C}=\mathrm{O}}=1729 ; v_{\mathrm{C}=\mathrm{O}}$ $=1696 ; v_{\mathrm{C}=\mathrm{O}}=1663 . \mathrm{MS} \mathrm{m} / \mathrm{z}(\%): 371\left(\mathrm{M}+\mathrm{H}^{+}, \mathrm{Na} ; 85\right)$. Anal. Calcd for $\mathrm{C}_{12} \mathrm{H}_{16} \mathrm{~N}_{2} \mathrm{O}_{5} \mathrm{Se}: \mathrm{C} 41.51$; H 4.64; N 8.07. Found: C 41.24; H 4.44; N 8.47.

Method B. The reaction was performed with 2-amino-1,3-selenazole $8(50 \mathrm{mg}, 0.16 \mathrm{mmol})$ and acetic anhydride $(51 \mathrm{mg}, 0.047 \mathrm{~mL}, 0.5 \mathrm{mmol})$. The work up of the reaction mixture was carried out as described for the synthesis of compound $\mathbf{4 a}$ to afford the title compound $\mathbf{9}$ (46 $\mathrm{mg}, 82 \%$ ).

\section{References and Notes}

1. (a) Koketsu, M.; Ishihara, H. 1,3-Selenazoles, In Comprehensive Heterocyclic Chemistry III; Katritzky, A. R.; Ramsden, C. A.; Scriven, E. F. V.; Taylor, R. J. K., Eds.; Elsevier: Oxford, 2008, Vol. 4; Chapter 4.08, pp 791-821. (b) Koketsu, M.; Ishihara, H. Current Org. Chem. 2003, 7, 175. (c) Mlochowski, J.; Kloc, K.; Lisiak, R.; Potaczek, P.; Wojtowicz, H. Arkivoc 2007, (vi), 14.

2. (a) Wray, S. K.; Smith, R. H. A.; Gilbert, B. E.; Knight, V. Antimicrob. Agents Chemother. 1986, 67. (b) Smee, D. F.; Huffman, J. H.; Hall, L. L.; Huggins, J. W.; Sidwell, R. W. Antiviral Chem. Chemother. 1990, 211.

3. Goot, van der, H.; Eriks, J. C.; Leurs, R.; Timmerman H. Bioorg. Med. Chem. Lett. 1994, 4, 1913.

4. (a) Kumar, Y.; Green, R.; Wise, D. S.; Wotring, L. L.; Townsend, L. B. J. Med. Chem. 1993, 36, 3849. (b) Kumar, Y.; Green, R.; Borysko, K. Z.; Wise, D. S.; Wotring, L. L.; Townsend, L. B. J. Med. Chem. 1993, 36, 3843. 
5. Sekiguchi, A.; Nishina, A.; Kimura, H.; Fukumoto, R.-H.; Kanoh, K.; Ishihara, H.; Koketsu, M. Chem. Pharm. Bull. 2005, 53, 1439.

6. Nam, K. N.; Koketsu, M.; Lee, E. H. Eur. J. Pharm. 2008, 589, 53.

7. (a) Keil, D.; Hartmann, H. Dyes and Pigments 2000, 44, 149. (b) Keil, D.; Hartmann, H.; Zug, I.; Schroder, A. J. Prakt. Chem. 2000, 42, 169.

8. (a) De Kimpe, N.; Verhe, R. The Chemistry of $\alpha$-Haloketones, $\alpha$-Haloaldehydes and $\alpha$-Haloimines; Patai, S.; Rappoport, Z., Eds.; Interscience: New York, 1988, pp 1-223. (b) Erian, A. W.; Sherif, S. M.; Gaber, H. M. Molecules 2003, 8, 793.

9. Geisler, K.; Pfeifer, W.-D.; Mueller, C.; Nobst, E.; Bulka, E.; Langer, P. Synthesis 2003, 1215.

10. (a) Narender, M.; Reddy, M. S.; Kumar, V. P.; Reddy, P.; Nageswar, Y. V. D.; Rao, K. R. J. Org. Chem. 2007, 72, 1849. (b) Potewar, T. M.; Ingale, S. A.; Srinivasan, K. V. Arkivoc 2008, (xii), 117.

11. Narender, M.; Majjigapu, S. R.; Vydyula, P. K.; Srinivas, B.; Shridhar, R.; Venkata, Y.; Nageswar, D.; Kakulapati, R. R. Synthesis 2007, 3469.

12. Bričkutè, D.; Sløk, F. A.; Šačkus, A. J. Chem. Soc., Perkin Trans. 1 2002, 652.

13. The CCDC deposition number of ethyl 2-(2-amino-5-ethoxycarbonyl-1,3-selenazol-4-yl)-2bromoethanoate (3) is 729374; formula: $\mathrm{C}_{10} \mathrm{H}_{13} \mathrm{BrN}_{2} \mathrm{O}_{4} \mathrm{Se}$; unit cell parameters: $a$ 8.2371(3), $b$ 18.5712(9), c 9.0273(3), $\beta$ 94.695(3); space group: P 21/c.

14. (a) Shi, X.; Zhao, G.-L. Acta Cryst. 2007, E63, 03642. (b) Al-Rubaie, A. Z.; Al-Masoudi, W. A.; Hameed, A. J.; Yousif, L. Z.; Graia, M. J. Korean Chem. Soc. 2008, 52, 36.

15. Socrates, G. Infrared Chracteristic Group Frequencies, $2^{\text {nd }}$ ed.; John Wiley and Sons: Chichester, New York, Brisbane, Toronto, 1994, p 58.

16. (a) Daroszewski, J.; Lusztyk, J.; Dequeil, M.; Navarro, C.; Maillard, B. J. Chem. Soc., Chem. Commun. 1991, 586. (b) Kim, K.-M.; Roberts, B. P. J. Chem. Research (S) 1998, 132. (c) Amoli, M.; Workentin, M. S.; Wayner, D. D. M. Tetrahedron Lett. 1995, 36, 3997.

17. (a) Altomare, A.; Cascarano, G.; Giacovazzo, C.; Guagliardi, A.; C.Burla, M.; Polidori, G.; Camalli, M. J. Appl. Cryst. 1994, 27, 435. (b) Mishnev, A. F.; Belyakov, S. V. Krystalligrafiya 1988, 33, 835 . 